\title{
Measurements of thermal photons in heavy ion collisions with PHENIX
}

\author{
T Dahms (for the PHENIX Collaboration) \\ Department of Physics and Astronomy, Stony Brook University, Stony Brook, \\ NY 11794-3800, USA \\ E-mail: torsten.dahms@stonybrook.edu
}

\begin{abstract}
Thermal photons are thought to be the ideal probe to measure the temperature of the quark-gluon plasma created in heavy ion collisions. PHENIX has measured direct photons with $p_{\mathrm{T}}<5 \mathrm{GeV} / c$ via their internal conversions into $e^{+} e^{-}$pairs in $\mathrm{Au}+\mathrm{Au}$ collisions at $\sqrt{s_{N N}}=200 \mathrm{GeV}$ and has now provided a baseline measurement from $p+p$ data.
\end{abstract}

PACS numbers: 25.75.Cj, 13.85.Qk, 12.38.Mh, 13.40.-f

\section{Introduction}

Direct photons are produced during all stages of heavy ion collisions at the Relativistic Heavy Ion Collider (RHIC). Because they do not interact strongly, they escape the medium unaffected by final state interactions and provide a promising signature of the earliest and hottest stage of the quark-gluon plasma (QGP) [1]. The main sources of direct photons from a QGP are quark-gluon Compton scattering $(q g \rightarrow \gamma q)$, quarkantiquark annihilation $(q \bar{q} \rightarrow \gamma g)$ and bremsstrahlung involving thermalized and incoming partons [2]. At RHIC energies thermal photons are predicted to be the dominant source of direct photons in $1<p_{\mathrm{T}}<3 \mathrm{GeV} / c$ [1].

The formation of a hot and dense medium has been established by experimental results at RHIC [3. The measurement with the Electromagnetic Calorimeter (EMCal), based on a statistical subtraction of the expected background form hadronic sources, remains limited at low $p_{\mathrm{T}}$ due to large systematic uncertainties [4, 5].

The uncertainties in the knowledge of the decay background can be reduced by avoiding its explicit measurement. A tagging method has been developed in which a very pure photon sample is selected with strict photon identification cuts [6]. In this sample those photons are tagged, which combined with other clusters in the EMC (selected with less stringent photon identification cuts) can be identified via their invariant mass as the result of a $\pi^{0}$ decay. In the ratio of pure photons and tagged photons the reconstruction efficiency of the pure photons cancel and with it the associated systematic uncertainty.

Furthermore, to circumvent the limitations due to the energy resolution at low photon energies, the excellent capabilities of the PHENIX detector to measure electrons can be utilized by measuring photons via their external conversion into $e^{+} e^{-}$ pairs [7. These conversion pairs can be used as an alternative clean photon sample in the method described above. 


\section{Internal Conversions}

To overcome the statistical and systematic limitations an alternative approach has been pursued [8] and is outlined in the following. Any process that produces a real photon can also create a virtual photon which converts internally into an $e^{+} e^{-}$pair. Therefore, $e^{+} e^{-}$pairs are also produced through internal conversions of virtual direct photons, e.g. via the $q g$ Compton scattering $\left(q g \rightarrow \gamma^{*} g \rightarrow e^{+} e^{-} g\right)$. The relation between photon production and the associated $e^{+} e^{-}$pair production can be written as:

$$
\frac{d^{2} n_{e e}}{d m}=\frac{2 \alpha}{3 \pi} \frac{1}{m} \sqrt{1-\frac{4 m_{e}^{2}}{m^{2}}}\left(1+\frac{2 m_{e}^{2}}{m^{2}}\right) S d n_{\gamma}
$$

with $m$ being the mass of the $e^{+} e^{-}$pair and $m_{e}=511 \mathrm{keV} / c^{2}$ the mass of the electron. The process dependent factor $S$ goes to 1 as $m \rightarrow 0$ or $m \ll p_{\mathrm{T}}$. For $\pi^{0}$ and $\eta$ decays, $S$ is given by $S=\left|F\left(m^{2}\right)\right|^{2}\left(1-m^{2} / m_{h}^{2}\right)^{3}[9]$ where $m_{h}$ is the hadron mass and $F\left(m^{2}\right)$ the form factor. For $e^{+} e^{-}$pair masses approaching $m_{h}$, the factor $S$ goes to zero. While the measurement of real thermal photons suffers from a large background of hadron decays, measuring virtual photons allows to select a mass range, in which $\pi^{0}$ decays are suppressed due to the cut-off in $S$.

The following analysis is based on $800 \mathrm{M}$ minimum bias events collected during the $\mathrm{Au}+\mathrm{Au}$ run at $\sqrt{s_{N N}}=200 \mathrm{GeV}$ in 2004 and $2.25 \mathrm{pb}^{-1}$ of single electron triggered data recorded during the $p+p$ run at $\sqrt{s}=200 \mathrm{GeV}$ in 2005. Electrons have been identified using information of the Ring Imaging Cherenkov detector (RICH) and a matching of momentum and the energy deposited in the EMC. Details of the analyses can be found in [10, 11]. The invariant mass spectrum of $e^{+} e^{-}$pairs after background subtraction is shown in Fig. [ 1 for $p+p$ (left) and $\mathrm{Au}+\mathrm{Au}$ (right) for various $e^{+} e^{-}$pair- $p_{\mathrm{T}}$ bins above $1 \mathrm{GeV} / c$. The data are corrected for the electron identification efficiency based on a full Monte Carlo simulation, but are not corrected for the geometrical acceptance [10, 11]. These data are then compared to a cocktail of hadron decays from a fast Monte Carlo simulation whose input is based on the measured hadron spectra [10, 11]. The cocktail is normalized to the data in the mass range $0-30 \mathrm{MeV} / c^{2}$. While in $p+p$ the agreement of the cocktail to the data is
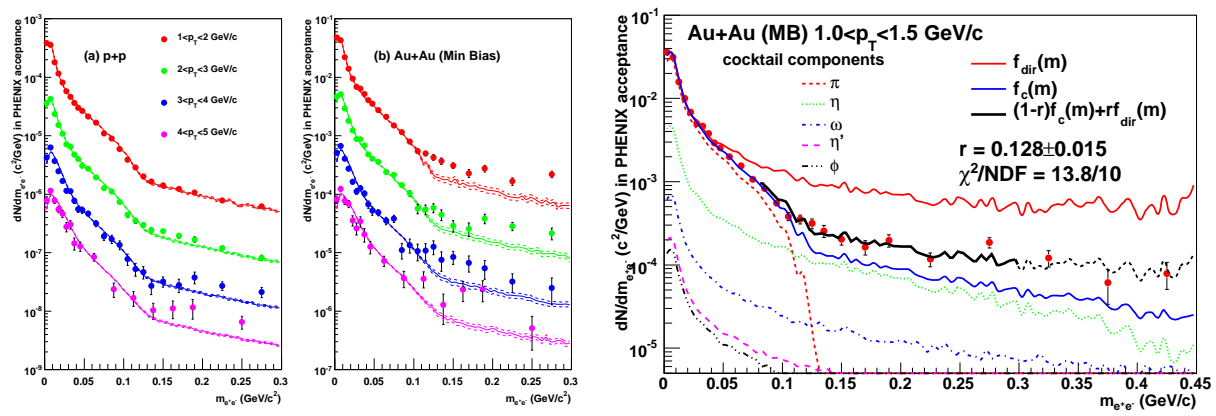

Figure 1. Left: The $e^{+} e^{-}$pair invariant mass distributions in (a) $p+p$ and (b) minimum bias $\mathrm{Au}+\mathrm{Au}$ collisions compared to a cocktail of hadronic sources. Right: Mass distribution of $e^{+} e^{-}$pairs for $\mathrm{Au}+\mathrm{Au}$ minimum bias events for $1.0<p_{\mathrm{T}}<1.5 \mathrm{GeV} / c$ with a fit as described in the text. 
remarkable and a small excess is only observed at very high $p_{\mathrm{T}}$ and large mass, the $\mathrm{Au}+\mathrm{Au}$ data show a large excess over the full $p_{\mathrm{T}}$ range of $1-5 \mathrm{GeV} / c$.

The observed excess above the hadronic cocktail can be analyzed under the assumption that it is solely due to internal conversions of direct virtual photons. While at zero mass $e^{+} e^{-}$pairs from hadron decays have the same shape in mass as internal conversions of direct photons, the suppression due to $S$ when approaching the hadron mass changes the shape of $e^{+} e^{-}$pairs from hadron decays. Therefore, one can fit, after an initial normalization to the mass range $0-30 \mathrm{MeV} / c^{2}$, the two expected shapes for $e^{+} e^{-}$pairs from hadronic decays $f_{\text {cocktail }}$ (as shown in Fig. 1) and from direct photons $f_{\text {direct }}$ in the mass range $80-300 \mathrm{MeV} / c^{2}$ in which the $\pi^{0}$ contribution is severely suppressed. The only fit parameter is the relative fraction of direct photons $r$ :

$$
f(m)=(1-r) f_{\text {cocktail }}(m)+r f_{\text {direct }}(m) .
$$

As an example, the fit result is shown in Fig. 1 for $e^{+} e^{-}$pairs with $1.0<p_{\mathrm{T}}<$ $1.5 \mathrm{GeV} / c$ in minimum bias $\mathrm{Au}+\mathrm{Au}$ collisions. The quality of the fit result (e.g., $\chi^{2} / N D F=13.8 / 10$ for the lowest $p_{\mathrm{T}}$ bin) indeed justifies the assumption that the observed excess is due to internal conversion of virtual photons. A fit with the mass shape of $e^{+} e^{-}$pairs from $\eta$ Dalitz decays would lead to a two times larger $\eta$ cross section than measured [12] and a significantly worse $\chi^{2} / N D F=21.1 / 10$. Little contribution from other sources is expected to this kinematic region, as it is limited to $m<2 m_{\pi}$. PHENIX has reported a large dielectron enhancement for $150<m<750 \mathrm{MeV} / c^{2}$ in [11, but the dominant fraction of the enhancement is concentrated at low $p_{\mathrm{T}}[13$.

\section{Results}

The result is shown as $1+r \approx \gamma_{\text {incl }} / \gamma_{\text {hadr }}$ in the left of Fig. 2 for $p+p$ collisions. It is compared to a NLO pQCD calculation of direct photons [14. While the fraction

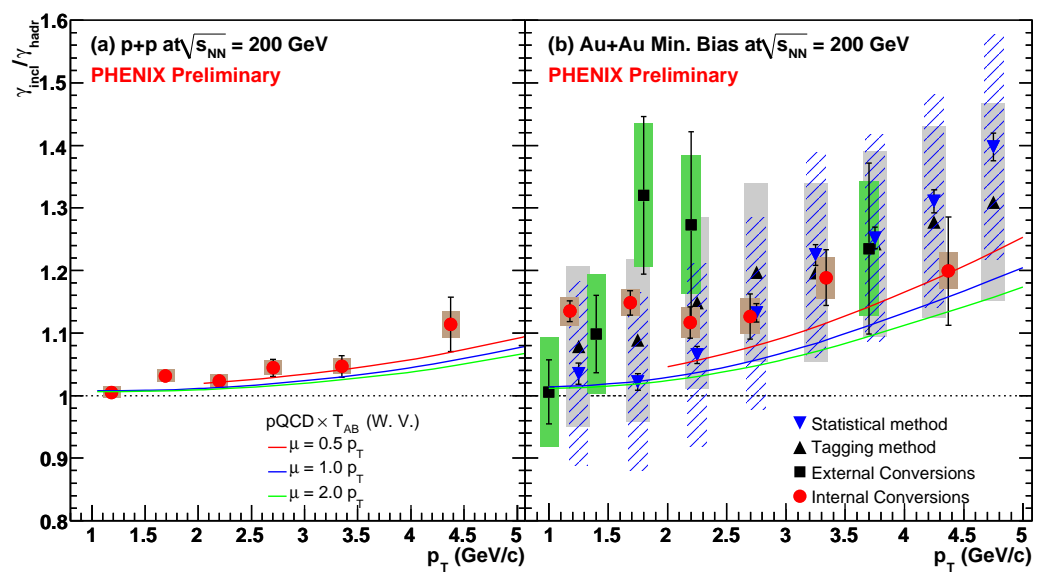

Figure 2. Direct photon excess. (a) The fraction of the direct photon component as a function of $p_{\mathrm{T}}$ in $p+p$. (b) $\mathrm{Au}+\mathrm{Au}$ (min. bias) compared to other measurements of direct photons as described in the legend. The curves are from a NLO pQCD calculation [14. 
of direct photons measured in $p+p$ agrees well with the pQCD calculation, a clear excess is observed in minimum bias $\mathrm{Au}+\mathrm{Au}$ collisions shown in the right of Fig. 2 $\mathrm{The} \mathrm{Au}+\mathrm{Au}$ result is also compared to the other measurements of direct photons at low $p_{\mathrm{T}}$; the conventional statistical subtraction of hadronic decay photons [15, the tagging method [6] and the external conversion analysis [7. While they all agree within their uncertainties, the improvement in statistical and systematic uncertainties is quite significant.

The fraction of direct photons $r$ can be converted into a direct photon spectrum by multiplying with the inclusive photon spectrum. The inclusive photon yield is determined for each $p_{\mathrm{T}}$ bin by $d N_{\gamma}^{\text {incl }}=d N_{e e}^{\text {data }} \times d N_{\gamma}^{\text {cocktail }} / d N_{e e}^{\text {cocktail }}$, where $d N_{e e}^{\text {data }}$ and $d N_{e e}^{\text {cocktail }}$ are the yields of $e^{+} e^{-}$pairs in $m<30 \mathrm{MeV} / c^{2}$ for data and cocktail, respectively, and $d N_{\gamma}^{\text {cocktail }}$ is the yield of photons from the cocktail. The resulting direct photon spectra for $p+p$ and $\mathrm{Au}+\mathrm{Au}$ collisions are shown in Fig. 3. The $p+p$ data can be well described with a modified powerlaw function: $A_{p p}\left(1+p_{\mathrm{T}}^{2} / b\right)^{-n}$. The direct photons measured in $\mathrm{Au}+\mathrm{Au}$ are above the binary scaled fit to the $p+p$ result. An exponential fit plus the binary scaled modified power law $\left(A \mathrm{e}^{-p_{\mathrm{T}} / T}+T_{A A} \times A_{p p}\left(1+p_{\mathrm{T}}^{2} / b\right)^{-n}\right)$ reveals an inverse slope of $T=220 \pm 23 \pm 18 \mathrm{MeV}$ for central collisions, suggesting an excess possibly of thermal origin. The inverse slope of the direct photon spectrum can be related to the intitial temparature of the QGP [16].

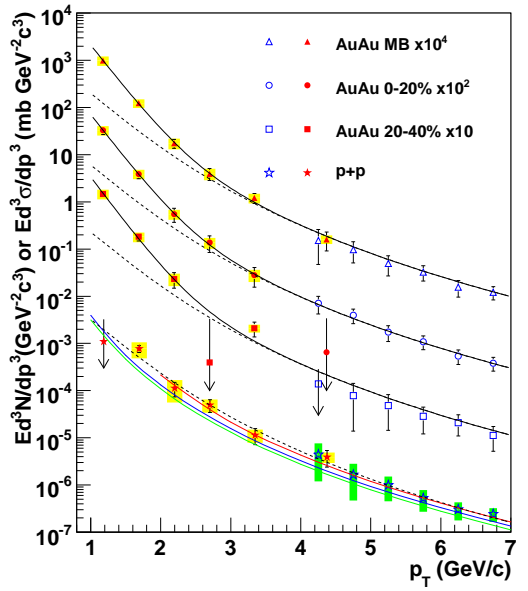

Figure 3. Invariant cross section $(p+p)$ and invariant yield $(\mathrm{Au}+\mathrm{Au})$ of direct photons. The $p+p$ result is compared to a NLO pQCD calculation. Solid points are from this analysis, open points from 4 [ 5 . The dashed lines show a $T_{A A}$ scaled, modified powerlaw fit of $p+p$. The black curves show an exponential plus the $T_{A A}$ scaled $p+p$ fit.

\section{References}

[1] Turbide S, Rapp R and Charles G 2004 Phys. Rev. C 69014903

[2] Aurenche P, Gelis F, Zaraket H and Kobes R 1998 Phys. Rev. D 8085003

[3] Adcox K et al. (PHENIX Collaboration) 2005 Nucl. Phys. A 757 184-283

[4] Adler S S et al. (PHENIX Collaboration) 2007 Phys. Rev. Lett. 98012002

[5] Adler S S et al. (PHENIX Collaboration) 2005 Phys. Rev. Lett. 94232301

[6] Gong H 2007 Preprint 0705.1133 [nucl-ex]

[7] Dahms T 2007 Proc. 23rd Winter Workshop on Nuclear Dynamics (EP Systema) p 1

[8] Adare A et al. (PHENIX Collaboration) 2008 Preprint 0804.4168 [nucl-ex]

[9] Landsberg L G 1985 Phys. Rept. 128 301-376

[10] Adare A et al. (PHENIX Collaboration) 2008 Preprint 0802.0050 [hep-ex]

[11] Afanasiev S et al. (PHENIX Collaboration) 2007 Preprint 0706.3034 [nucl-ex]

[12] Adler S S et al. (PHENIX Collaboration) 2006 Phys. Rev. Lett. 96202301

[13] Toia A 2008 these Proceedings

[14] Gordon L E and Vogelsang W 1993 Phys. Rev. D 48; and private communication

[15] Isobe T 2007 J. Phys. G: Nucl. Part. Phys. 34 S1015-S1018

[16] d'Enterria D and Peressounko D 2006 Eur. Phys. J. C 46 451-464 